\title{
Exciton states in two-dimensional systems of GaAs/AlAs multi-quantum-well structures under high magnetic fields
}

\author{
T. Yasui and Y. Segawa \\ Photodynamics Research Center, Frontier Research Program, \\ The Institute of Physical and Chemical Research (RIKEN), 19-1399 Koeji, Nagamachi, Aoba, Sendai, 980, Japan \\ Y. Aoyagi \\ The Institute of Physical and Chemical Research (RIKEN), Saitama, Japan \\ Y. Iimura \\ Tokyo University of Agriculture and Technology, Tokyo, Japan \\ G. E. W. Bauer \\ Delft University of Technology, Delft, The Netherlands \\ I. Mogi \\ Institute for Materials Research, Tohoku University, Sendai, Japan \\ G. Kido \\ Tsukuba Magnet Laboratories, National Research Institute for Metals, Tsukuba, Japan
}

(Received 29 August 1994)

\begin{abstract}
Magneto-optical spectra of a GaAs/AlAs multi-quantum-well sample have been measured in the Faraday configuration at high magnetic fields up to $25 \mathrm{~T}$. These spectra reveal clear excitonic effects on top of the Landau-level structure. The excitonic states are well explained by effective-mass calculations that take into account residual electric fields in the sample and the valence-band mixing in magnetic fields. The results indicate that the Coulomb interaction plays an important role even under very high magnetic fields, in contrast to the common belief that it should be only a weak perturbation to the Landau level. A crossing of the lowest heavy-hole free exciton and the lowest light-hole free exciton is observed at a magnetic field of about $15 \mathrm{~T}$ with $\sigma^{+}$polarization, thus achieving a symmetry change in the exciton ground state. The absence of an anticrossing between the light- and heavy-hole exciton ground state indicates the unimportance of exchange effects.
\end{abstract}

\section{INTRODUCTION}

To describe the behavior of carriers in quantum wells (QW's) under high magnetic fields, we have to consider perturbations, such as confinement, magnetic fields, Coulomb interactions between electron and hole, and impurity states or defects in the sample. Two-dimensional exciton states confined in QW's have been studied by many researchers since the early report by Dingle, Wiegmann, and Henry, ${ }^{1}$ Miller et al., ${ }^{2}$ Bastard et al., ${ }^{3}$ Green, Bajai, and Phelps, ${ }^{4}$ Matsuura and Shinozuka, ${ }^{5}$ and Miller and Kleinman. ${ }^{6}$ As for GaAs/AlAs quantum wells, the symmetry point group is changed along the axis normal to the well because of the broken translational invariance in the growth direction. The symmetry change split the top of the valence band into heavy and light holes. In addition, the quantum size effect produces subband levels in the conduction and valence band. ${ }^{1}$ Exciton states are generated between conduction electron subband levels $(n=1,2,3, \ldots)$ and valence hole subband levels. In the absence of magnetic fields, hydrogenic states $(1 s, 2 s, 2 p, 3 d, \ldots)$ of excitons can be expected because of the Coulomb interaction between electron and hole. The
$2 s$ (Ref. 7) and $3 s$ (Ref. 8) states of the heavy-hole free exciton in GaAs QW's were reported, but fine structure representing the higher states of the heavy- (light-) hole excitons has not been found, owing to the weak oscillator strength of the exciton higher states and due to the carrier scattering factors depending on the sample quality. Optical measurement in high magnetic fields is a useful method against this difficulty, since discrete energy lev$\mathrm{els}^{9-11}$ and enhancement of the oscillator strength of excitons ${ }^{12-15}$ is induced by magnetic fields. Accurate measurements under high magnetic fields give information on the fine structure of the spectra. On the other hand, magnetic fields cause an external perturbation in addition to the confinement effect. Thus, these measurements also provide new problems concerning the behavior of carriers in the samples, as described below.

The first studies of excitons in QW's under high magnetic fields were focused on the diamagnetic shifts of the lowest exciton states, ${ }^{9,10}$ which stimulated other theoretical and experimental study on this problem. ${ }^{16,20}$ Peaks in the spectra at higher photon energies were interpreted as Landau level transitions in the early experimental reports. $^{9,11}$ In this point of view, exciton states are dom-

\footnotetext{
9813 (c) 1995 The American Physical Society
} 
inant in the weak magnetic-field range and the excitonic nature should vanish in high magnetic fields, since the external perturbation of the excitons by the magnetic fields will overcome the Coulomb interaction between the electron and hole.

Contrary to this interpretation, the theory predicted that excitonic states exist also at high magnetic fields. ${ }^{21-28}$ In spite of the theoretical prediction, good agreement with the experimental results was not always obtained. ${ }^{25}$ Good agreement between experiment ${ }^{12}$ and the theory ${ }^{26}$ was obtained only later by using high-quality samples and more careful calculations. In recent years, the behavior of magnetoexcitons has been studied more precisely under intermediate ${ }^{13}$ and high magnetic fields. ${ }^{14,15}$ These studies have shown that the magnetoexcitons in GaAs/AlAs quantum wells reflect the complicated valence-band structure and have a complicated behavior under high magnetic fields. MacDonald and Ritchie have discussed unmixed magnetoexciton states in weak and strong magnetic fields. ${ }^{24}$ However, theory can predict the behavior of the magnetoexcitons well only by considering exciton mixing. ${ }^{27}$

The problem here is the different interpretations of the optical spectra of a GaAs QW under high magnetic fields. That is to say, whether the decrease in the relative importance of the excitonic effects with magnetic field is showing enough to explain experiments in a Landau-level model. What seems to be lacking in the previous magneto-optical studies ${ }^{9-11,13-15}$ is the discussion concerning the relation between the Landau-level model and magnetoexcitons. This problem reflects the difficulty of dealing with all factors: magnetic fields, Coulomb interaction, the quantum size effects, and nonradiative process depending on the sample quality.

In this work, to further explore the above problems, we report the results of photoluminescence excitation (PLE) spectra in the same sample used in an earlier work, ${ }^{13}$ but extend the applied magnetic field up to $24 \mathrm{~T}$. The results are also compared with the theory. ${ }^{27}$ Due to the higher magnetic fields, the mixed perturbation described above for the carriers in QW's will be discussed. The paper is organized as follows: In Sec. II, we present the details of experiment. In Sec. III, we demonstrate the interpretation for the experimental results by the magnetoexciton theory. We also discuss that our results differ from a simple Landau-level model. Finally, in Sec IV, our results are summarized.

\section{EXPERIMENT}

The sample used in this work is a multi-quantum-well structure with 40 periods of $265-\AA-G a A s$ wells and $260-$ $\AA$-AlAs barriers, the details of which are reported elsewhere. ${ }^{13}$ The experimental setup is shown in Fig. 1. High magnetic fields up to $25 \mathrm{~T}$ were generated by a hybrid magnet at the High Field Laboratory for Superconducting Materials in Tohoku University. The sample was placed in a hybrid magnet consisting of an outer superconducting magnet and an inner water-cooled resistive magnet. The sample temperature was kept at $4.2 \mathrm{~K}$ by immersion in liquid helium, as indicated by the dotted

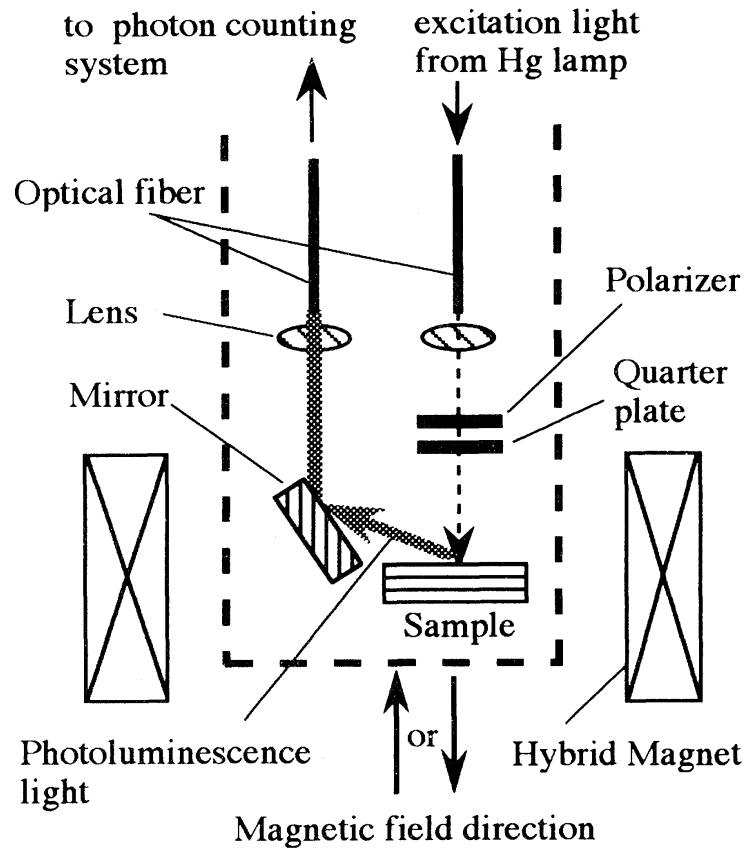

FIG. 1. Experimental setup for the PLE spectroscopy using the optical fiber to lead the excitation light into the hybrid magnet and to detect the signal light.

line in Fig. 1. The magnetic fields in this study ranged from 6 to 25 T.PLE spectra were obtained by monitoring the luminescence of the lowest free-exciton level when the excitation light was scanned. The observed intensities of the ground state are proportional to the oscillator strengths of the excited states, while the spectra include the information on the relaxation process of the photoexcited carriers. Then PLE spectra correspond to the absorption spectra. Light from a $250-\mathrm{W}$ mercury lamp with a flat band in the spectral region of this study was passed through a monochromator with a spectral resolution of about $1 \mathrm{meV}$. The incident light was circularly polarized in front of the sample so as to make a Faraday configuration with $\sigma^{+}$and $\sigma^{-}$polarization. The emitted light was detected by another monochromator at the photon energy of the lowest exciton level included both spin up and spin down and led to a conventional photon counting system.

\section{RESULTS AND DISCUSSION}

\section{A. PLE spectra in high magnetic fields}

Figures 2(a) and 2(b) show some of the $\sigma^{+}$and $\sigma^{-}$ PLE spectra at various magnetic-field strengths in the photon-energy region from 1.52 to $1.58 \mathrm{eV}$. Most of the peaks in these spectra were given temporary labels $(A, B$, $C$, etc.) and assigned to exciton states according to calculations described later. We use the following notations for peaks: $H(L)$ for heavy (light) holes; the first number is the electron subband and the second is the hole subband; and a hydrogenic $(\mathrm{nm})$ is used for the exciton envelope functions, where $n$ is the principal quantum num- 

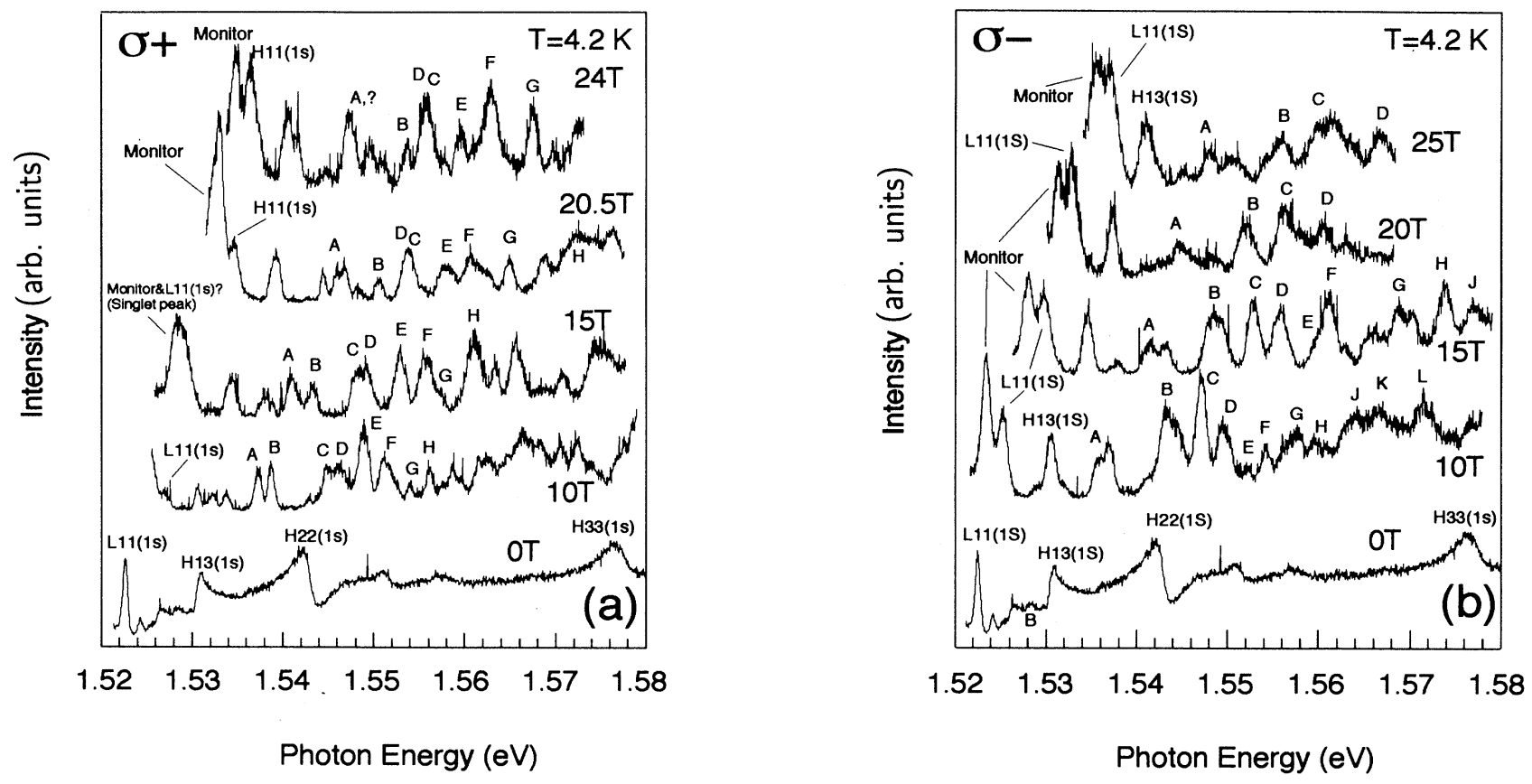

FIG. 2. (a) and (b) PLE spectra of a 265- $\AA$-width GaAs well sample at various magnetic fields with $\sigma^{+}$and $\sigma^{-}$polarization, respectively. Peaks with temporary labels $(A, B, C, \ldots$ etc.) are assigned to exciton levels based on calculations from earlier reports (Ref. 27). Other peaks were labeled as mentioned in the text. Note that $L 11(1 s)$ and the monitor peak become the singlet peak at 15 T only with $\sigma^{+}$polarization. The details are mentioned in the text.

ber and $m$ is the angular-momentum quantum number $\left(\ldots d^{-}, p^{-}, s, p^{+}, d^{+}, \ldots\right)$. The spin labels are used, if necessary. To avoid complexity, this labeling system is limited to only a few cases in Figs. 2(a) and 2(b). At $0 \mathrm{~T}$, some peaks corresponded to exciton states are seen as indicated by $L 11(1 s), H 13(1 s), \ldots$ and so on. The higher states up to the $n=3$ heavy-hole subband level are included in this photon-energy region at $0 \mathrm{~T}$. Compared to the zero-field spectra both of $\sigma^{+}$and $\sigma^{-}$polarization, many discrete peaks are clearly revealed and the background level decreases as the magnetic-field strength increases. These changes can be explained by the effects of the magnetic fields, that is to say, both the increase in the oscillator strength with reduction of the exciton radius and the quantization of the density of states. The energy shifts of these peaks are also observed as the magnetic field increases. In Fig. 2(a) for $\sigma^{+}$polarization, one peak observed as $L 11(1 s)$ is in the higher energy side of the monitor peak $[=H 11(1 s)]$ under $10 \mathrm{~T}$. The two peaks become a singular peak at $15 \mathrm{~T}$ and split again into two peaks over $20 \mathrm{~T}$. In $\sigma^{-}$case, however, this behavior cannot be observed and the $L 11(1 s)$ peak is clearly observed in these magnetic-fields region. These characters indicate that $H 11(1 s)$ and $L 11(1 s)$ states cross at $15 \mathrm{~T}$ only in the $\sigma^{+}$polarization. But, it is difficult to show the exact evidence of the crossing, since the monitor peak in PLE spectra is the ground state $[=H 11(1 s)]$. The detailed discussions of this point are mentioned in Sec. III C. Another noticeable point is that several peaks are observed at over $20 \mathrm{~T}$, even in the photon-energy region close to the $H 11(1 s)$ exciton. It is obvious that a simple Landau-level model cannot well explain this behavior. To clarify these points, we shall focus on the energy shifts of these peaks and the changes in the oscillator strength of excitons as a function of magnetic field.

\section{B. Comparison with the Landau-level model and the magnetoexciton model}

The energy shifts in excitons for $\sigma^{+}$and $\sigma^{-}$are shown as curves in Fig. 3. The oscillator strengths are proportional to the area of the circles. The lowest states as monitored peaks are indicated by the solid curves. The oscillator strengths are normalized to $H 12(1 s)$, which was constant throughout this measurement. Complicated energy shifts can be observed below $11 \mathrm{~T}$ in both Figs. 3(a) and 3(b). On the other hand, this behavior is simplified in the high magnetic-field region, where most peaks show a linear energy shift. We attempt to compare these results with Landau-level transitions in Figs. 4(a) and 4(b) for $\sigma^{+}$and $\sigma^{-}$. The PLE spectra are for various magnetic fields above $10 \mathrm{~T}$. The calculated interLandau-level transitions (limited to $n=0,1,2$ ) are indicated by the broken lines in each spectra. These Landaulevel transition energies $(=\Delta E)$ were deduced from the relation

$$
\Delta E=E_{\mathrm{sub}}+\Delta E_{e}+\Delta E_{h} .
$$

Here, $E_{\text {sub }}$ is the calculated inter-subband energy $(=1.52714 \mathrm{eV})$ of our sample between the first subband 

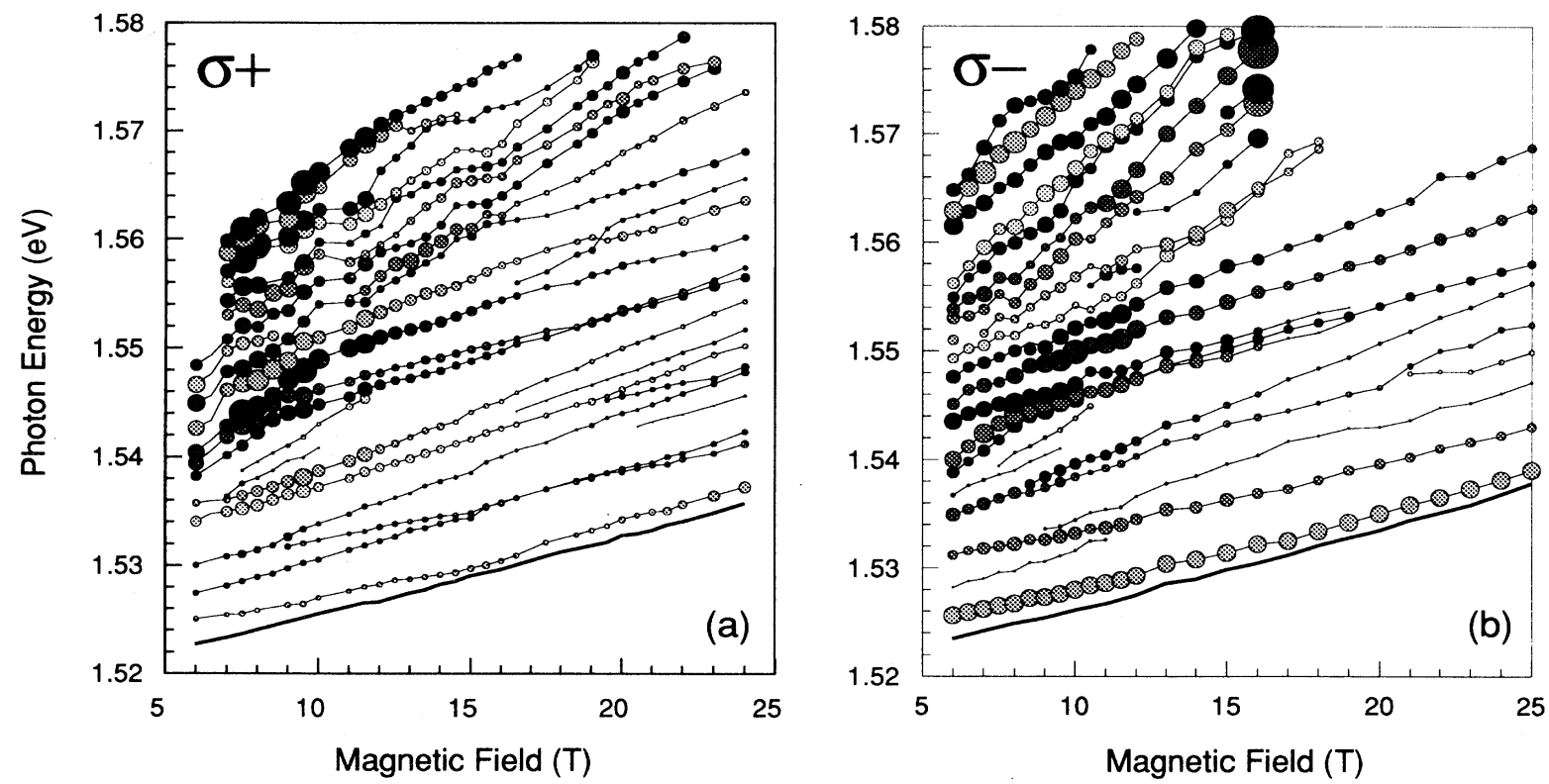

FIG. 3. Energy shifts of peaks in PLE spectra as a function of magnetic field up to $25 \mathrm{~T}$ with $\sigma^{+}$polarization, (a), and $\sigma^{-}$polarization, (b). The solid curves correspond to the lowest exciton state, and are used as the monitor peak. The area of the circles are proportional to the oscillator strengths of peaks in PLE spectra.
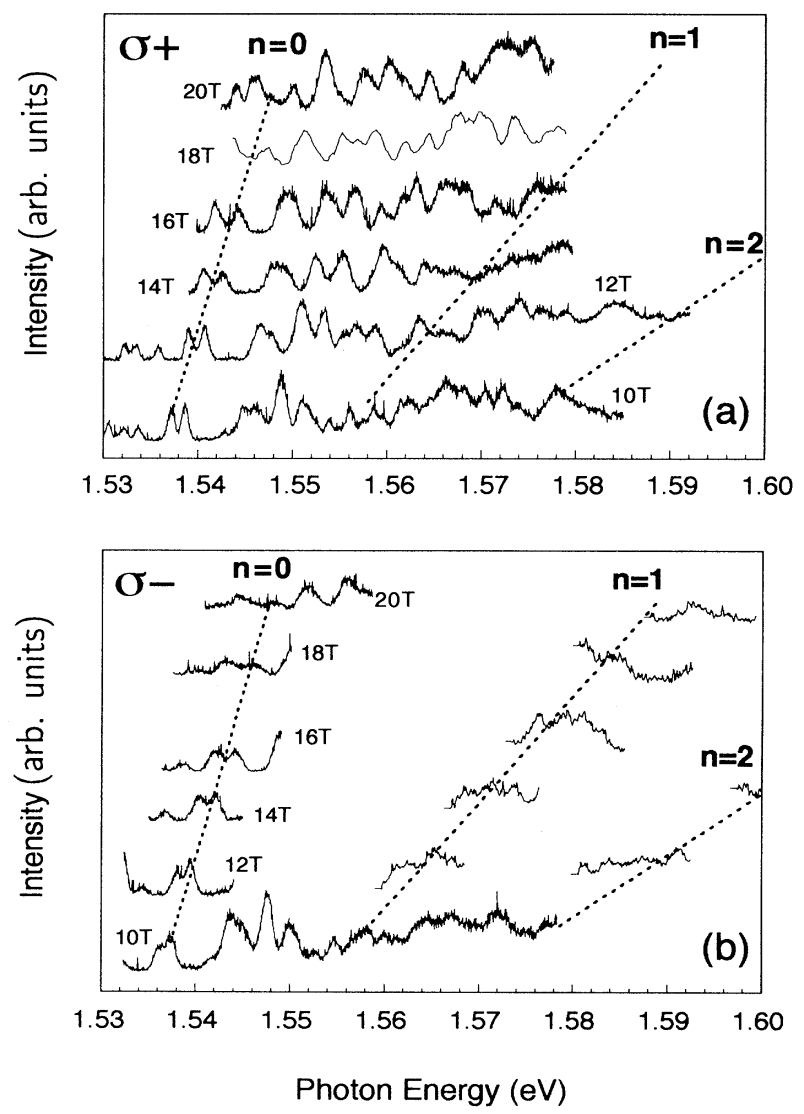

FIG. 4. (a) and (b) Comparison with PLE spectra and Landau-level transitions above $10 \mathrm{~T}$. PLE spectra were measured for $\sigma^{+}$(top) and $\sigma^{-}$(bottom) polarization at $4.2 \mathrm{~K}$. The energy of Landau-level transitions for $n=0,1$, and 2 are indicated by the broken lines. of conduction and that of the valence band. ${ }^{27}$ The $\Delta_{e}$ ( $\Delta E_{h}$ ) is the cyclotron energy of the free electrons (holes) as given by

$$
\begin{aligned}
& \Delta E_{e}=\frac{h e B}{2 \pi m_{e}}\left(n+\frac{1}{2}\right), \quad m_{e}=0.067 m_{0}, \\
& \Delta E_{h}=\frac{h e B}{2 \pi m_{h}}\left(n+\frac{1}{2}\right), \quad m_{h}=0.35 m_{0},
\end{aligned}
$$

where $m_{e}\left(m_{h}\right)$ is the effective mass of the free electrons (holes) in GaAs as written above, and $B$ is the magneticfield strength. The other symbols have the usual meanings. Inter-Landau-level transitions show a linear dependence on the magnetic fields. In Fig. 4, however, the behaviors of all peaks in the spectra differ from the Landau-level transitions in every respect: photon energy, magnetic-field dependence, and polarization of excitation light. The Landau-level nature was not seen, even under the magnetic fields of up to $24 \mathrm{~T}$.

Therefore, we tried to explain our results with the magnetoexciton theory, ${ }^{27}$ as shown in Fig. 5 for $\sigma^{+}$and Fig. 6 for $\sigma^{-}$. The bold curves are the experimental results for lowest exciton states, but are theoretical results for $H 11(1 s)$. This difference is due to the crossing behavior seen in Fig. 5(a), which is discussed later. The details of the calculations are given in Ref. 27. The calculations, which are based on effective-mass theory, take into account the complicated valence-band structure, the effects of a residual electric field in the sample, and magnetic fields. The exciton labels in these figures indicate the dominant exciton component. Unlabeled peaks indicate that many components of equal importance coexist in the same level. Forbidden transitions, which are marked with asterisks in Fig. 6(a), become observable due to the residual electric fields in our sample. Earlier work es- 


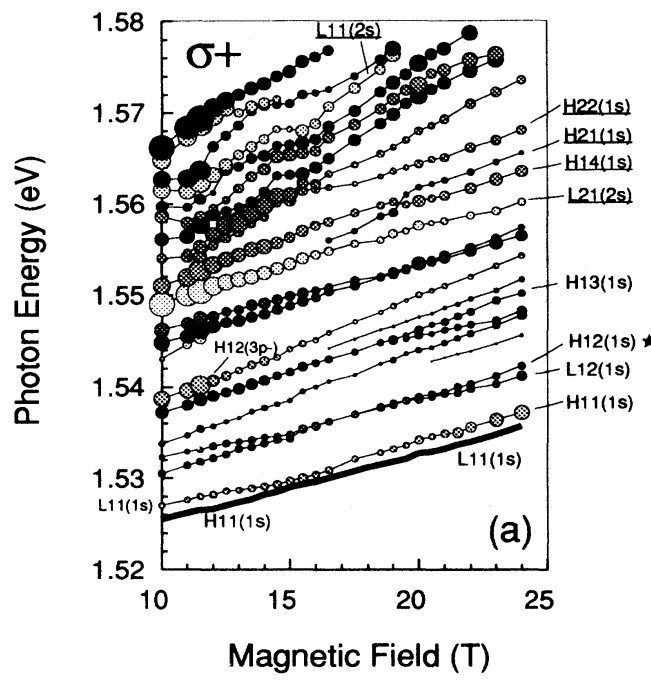

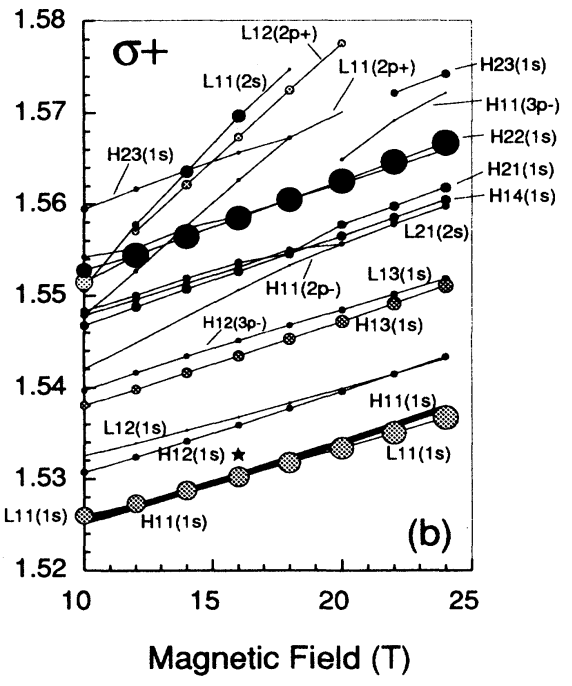

FIG. 5. Comparison with experiment (a) and calculations (b) for $\sigma^{+}$polarization. Energy shifts and oscillator strengths of exciton levels are plotted vs magnetic-field strengths. The circles and the curves have the same meaning as in Fig. 3. Exciton labels mean the dominant exciton component of peaks. The unlabeled peaks indicate that multiple dominant components coexist in the same peak. The underlined peaks indicate uncertain assignments. The asterisk $(*)$ is added for forbidden transitions, which can be observed because of the residual electric field in the sample. timated the value of the residual electric field to be about $3 \mathrm{kV} / \mathrm{cm}{ }^{13}$ This residual electric field is caused by the Fermi-level pinning at an interface between the GaAs layer and the AlAs layer. The band symmetry of the QW is broken in such a system and some of the forbidden transitions are optically allowed. A few states mix with each other (see from 1.54 to $1.55 \mathrm{eV}$ ) below $11 \mathrm{~T}$. The oscillator strengths of these peaks show very complicated changes. These features are clear evidence for the excitonic nature. In contrast to this, as mentioned above, the spectra are simpler in the high magnetic-field region. Some disagreement between experiment and theory is noted for the higher states of excitons under high magnetic fields, as indicated by the underlined labels in Figs. 5(a) and 6(a). This is assumed to be due to the basis set limitations in the calculations. In other words, the basis for the radial functions in the plane of the well can per- form satisfactorily only for zero or intermediate magnetic fields (below $10 \mathrm{~T}$ ). ${ }^{27}$ Even if some disagreement remains, it is clear that the excitonic effects in our study of a 265A-thick GaAs well remain significant even for high magnetic fields.

\section{A symmetry change in the exciton ground state}

As it is already stated in the previous paragraph, for the $\sigma^{+}$polarization the crossing of $H 11(1 s)$ and $L 11(1 s)$ at $15 \mathrm{~T}$ is observed in Fig. 5(a), but not in Fig. 6(a). Figure 7 depicts the $\sigma^{+}$PLE spectra around the monitor peak from 10 up to $24 \mathrm{~T}$. Figures 5(a) and 7, however, do not show exact evidence of the crossing behavior, because the lowest exciton state (closed or open circles in Fig. 7) is used as the monitoring peak (closed triangles in Fig. 7) in these measurements. This is because it is impossible to

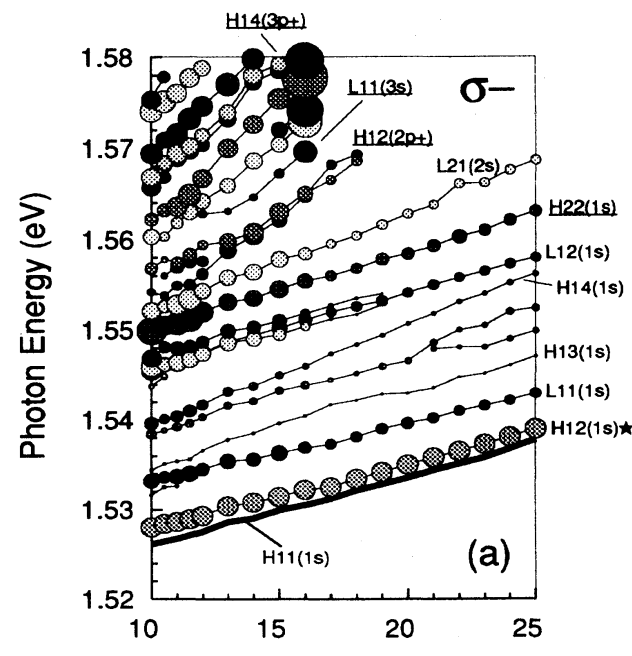

Magnetic field (T)

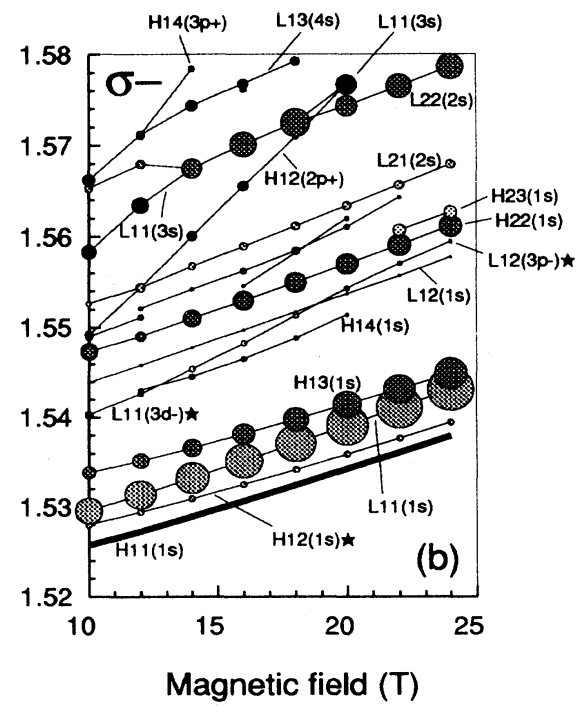

FIG. 6. Comparison with experiment (a) and calculations (b) for $\sigma^{-}$polarization. Otherwise, these figures are same as Fig. 5. 


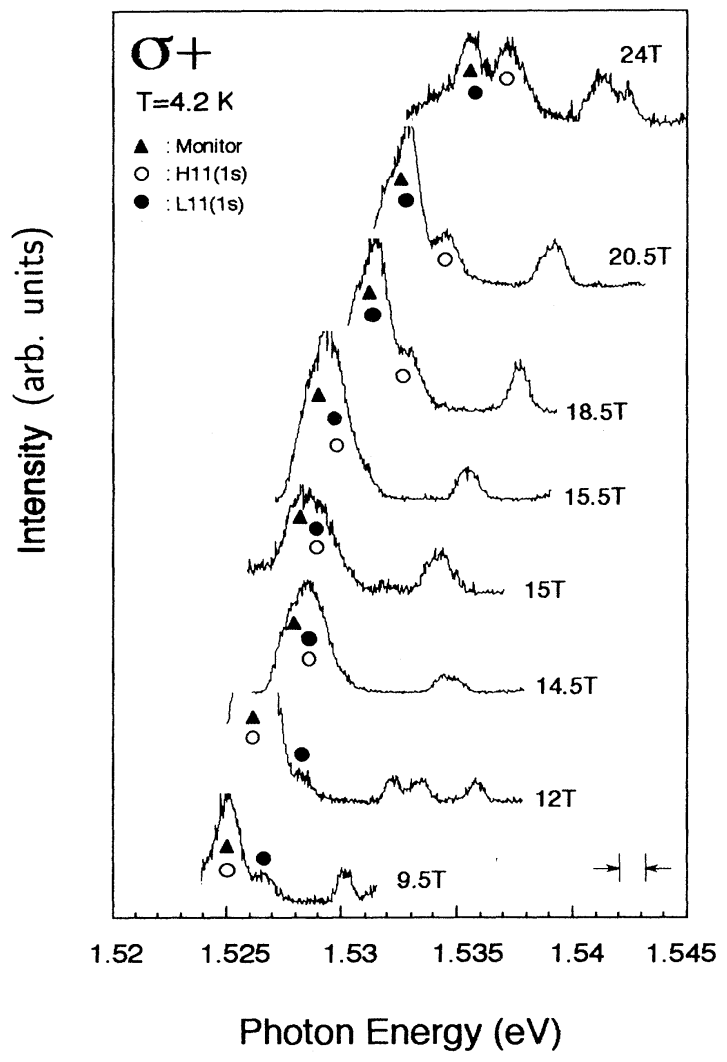

FIG. 7. The $\sigma^{+}$PLE spectra limited to the photon-energy region around the monitor peak. This figure depicts a part of Fig. 2 (a) by the detailed magnetic-field steps. The symbols $(\boldsymbol{\Delta}, \bigcirc, \mathbf{O})$ used in this figure have the following meanings: closed triangles $(\Delta)$ are the monitoring energy positions; open circles $(O)$ are the H11(1s); and (O) closed circles are the $L 11(1 \mathrm{~s})$. The exciton ground state ( $O$ or $\bigcirc$ ) is monitored in this measurement.

discriminate between the lowest exciton and the monitor peak. To clarify this point, Fig. 8 shows the detailed PLE spectra measured by monitoring the energy position shifted to a lower value (about $0.3 \mathrm{meV}$ ) from the lowest exciton states. This method differs from the conventional PLE measurement and makes it possible to observe the energy position of the monitor peak and the lowest state, respectively. Figure 8 shows $H 11(1 s)$ and $L 11(1 s)$ close to each other at around $15 \mathrm{~T}$. Since $H 11(1 \mathrm{~s})$ belongs to $\Gamma_{7}$ of the symmetry point group and $L 11(1 s)$ belongs to $\Gamma_{6}$ of the symmetry point group, these two states are allowed to cross only when exchange effects are negligible. It is obvious that the lowest exciton state is replaced by $L 11(1 s)$ at above $15 \mathrm{~T}$ for the $\sigma^{+}$polarization. The spin splitting energy of $H 11(1 s)$ is smaller than that of $L 11(1 s)$, so it is difficult to observe in PLE measurement. Therefore, the $H 11(1 s)$ peak crossed $L 11(1 s)$ in the $\sigma^{+}$ configuration, but not in the $\sigma^{-}$configuration. This crossing is well explained by the calculations, as shown in Fig. 5(b).

The crossing causes the final relaxation state to change into $L 11(1 s)$ in the PLE measurement. Since the photoluminescence efficiency reflects this change in the relaxation process, the intensities of almost all peaks decrease in Fig. 5(a), for $\sigma^{+}$polarization above $15 \mathrm{~T}$, where $L 11(1 s)$ is the monitoring peak. This change in signal intensity is not as remarkable in Fig. 6(a) for the $\sigma^{-}$polarization, as in Fig. 5(a), because no change of the symmetry point group of the lowest exciton state is found.

\section{The magnetoexciton states in GaAs QW}

These exciton states in high magnetic fields have been observed for GaAs well thicknesses of $160,{ }^{14} 45,{ }^{15}$ and 30 $\AA .{ }^{15}$ More complicated spectra can be observed by increasing the well thickness by Kusano, Bauer, and Aoyagi. ${ }^{29}$ In these reports, the magnetoexciton states offer a good explanation of the experimental results as well as our results in Figs. 5 and 6. Some disagreement between experimental results and the calculations based on the magnetoexciton model have been noted for high magnetic fields in our work and in the other study. ${ }^{14}$ This disagreement indicates the difficulty of solving the problem of the transitional state from weak magnetic fields to the strong ones, where we should consider a complex system of mixed perturbations for the carriers in the sample. That is to say, the magnetic field as an one-dimensional perturbation, Coulomb interaction as a three-dimensional perturbation, and the confinement effect as a twodimensional perturbation are all effective for the carriers
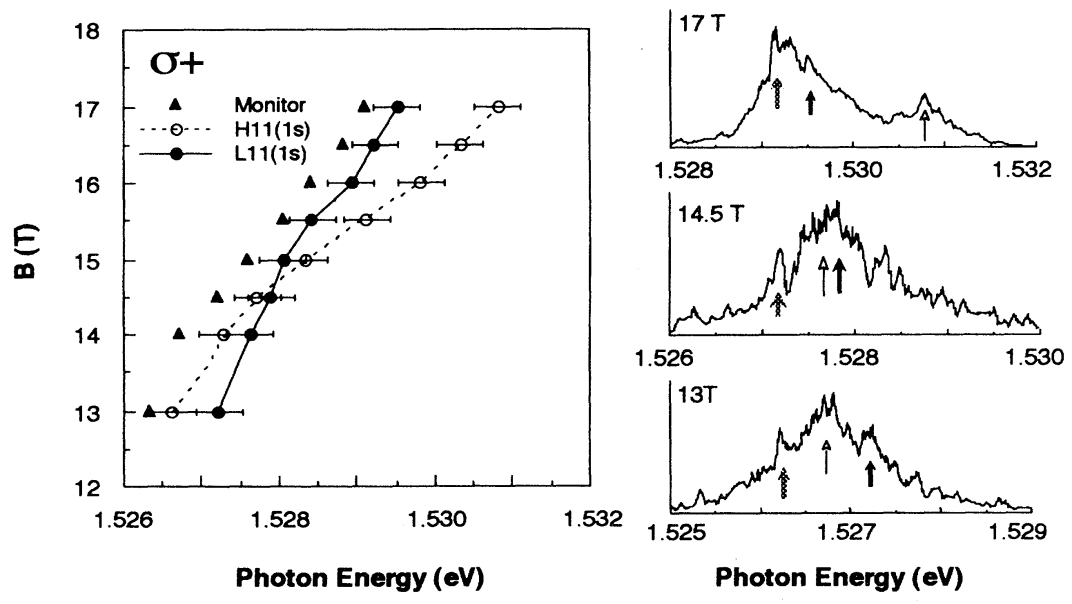

FIG. 8. The crossing of $H 11(1 s)$ and $L 11(1 s)$ for $\sigma^{+}$polarization. The PLE spectra at $13,14.5$, and $17 \mathrm{~T}$ are shown above right. The symbols $(\boldsymbol{\Lambda}, \bigcirc, \Theta)$ have the same meanings as in Fig. 7. These PLE spectra were measured by monitoring the energy position ( $\boldsymbol{\Delta}$ ), which was shifted toward lower energies of the lowest exciton states ( $O$ or $\bigcirc$ ). Magnetic fields vs photon energy of the monitored peak, $H 11(1 s)$ and $L 11(1 s)$ are plotted above left. The error bars indicate the energy resolution overlap at $14-15 \mathrm{~T}$. $H 11(1 s)$ and $L 11(1 s)$ become a singlet peak, as shown in the PLE spectra at $14.5 \mathrm{~T}$, where it indicates the crossing behavior. 
in the QW sample. Added to this, the residual electric field must be considered in case of our sample as mentioned above. We expected to be able to discuss the relationship between the dimensionality of the external perturbation and the behavior of the carriers in the sample, but it is difficult to present the whole discussion about this point below $25 \mathrm{~T}$. Magneto-optical measurements above $25 \mathrm{~T}$ will provide a basis for fruitful discussion about the dimensionality of the external perturbation for the carriers in QW under high magnetic fields.

On the other hand, the Landau-level model gave a reasonable explanation of the energy shifts in the first magneto-optical study for GaAs quantum wells, where the well thickness region is 50 to $125 \AA$ (Ref. 9) and 58 to $252 \AA{ }^{10}$ It is difficult to identify the exact cause of this difference from our experimental results because we had not compared with other samples which Landau-level nature is dominant. We, however, assume that the difference occurs because free carriers of electrons (or holes) are originated from the unintentionally doped carriers in the sample and from the ionization of excitons due to scattering by the impurities, etc. When the difference is exhibited clearly by the sample quality, the number of free carriers overcome the number of excitons and the free carriers mainly compose Landau-level states in high magnetic fields. Contrary to this, Coulomb interaction to make exciton states is more sensitive for the sample quality depending on defects, impurities, and so on since the existence of impurities intercarriers will in- tercept the Coulomb interaction inside the excitons. This is remarkable for the more excited states of excitons with the extended exciton wave function. This is because the observation of excitonic nature in high magnetic fields needs the high quality of the samples. In all these cases, the sample quality has an important role on the number of free carriers and on the exciton lifetime. We think that these factors are the reason why the excitonic states are observed only in the high quality samples and the Landau-level states are dominant in other samples.

\section{CONCLUSION}

In conclusion, we have shown hydrogenlike exciton states under high magnetic fields by magneto-optical studies of a 265 -A-thick GaAs quantum well. The experimental results cannot be explained by a simple Landaulevel model. The excitonic nature is confirmed by effective-mass calculations, which take into account valence-band mixing and external perturbations. Coulomb interaction in excitons should be considered as an important effect in explaining the behavior of the carriers in QW under high magnetic fields below $25 \mathrm{~T}$. The crossing of the lowest exciton states is observed for the $\sigma^{+}$polarization at around $15 \mathrm{~T}$, where it indicates a symmetry change of the exciton ground state. The absence of an anticrossing between these states proves the unimportance of the exchange interaction in GaAs quantum wells.
${ }^{1}$ R. Dingle, W. Wiegmann, and C. H. Henry, Phys. Rev. Lett. 33, 827 (1974).

${ }^{2}$ R. C. Miller, D. A. Kleinman, W. T. Tsang, and A. C. Gossard, Phys. Rev. B 24, 1134 (1981).

${ }^{3}$ G. Bastard, E. E. Mendez, L. L. Chang, and L. Esaki, Phys. Rev. B 26, 1974 (1982).

${ }^{4}$ Ronald L. Green, Krishan K. Bajai, and Dwight E. Phelps, Phys. Rev. B 29, 1807 (1984).

${ }^{5}$ Mitsuru Matsuura and Yuzo Shinozuka, J. Phys. Soc. Jpn. 53, 3138 (1984).

${ }^{6}$ R. C. Miller and D. A. Kleinman, J. Lumin. 30, 520 (1985).

${ }^{7}$ K. J. Moore, P. Dawson, and C. T. Foxon, Phys. Rev. B 34, 6022 (1986).

${ }^{8}$ D. C. Reynolds, K. K. Bajaj, C. Leak, G. Peters, W. Theis, P. W. Yu, K. Alavi, C. Colvard, and I. Shidlovsky, Phys. Rev. B 37, 3117 (1988).

${ }^{9}$ J. C. Maan, G. Belle, A. Fasolino, M. Altarelli, and K. Ploog, Phys. Rev. B 30, 2253 (1984).

${ }^{10}$ S. Tarucha, H. Okamoto, Y. Iwasa, and N. Miura, Solid State Commun. 52, 815 (1984).

${ }^{11}$ D. C. Rogers, J. Singleton, R. J. Nicholas, C. T. Foxon, and K. Woodbridge, Phys. Rev. B 34, 4002 (1986).

${ }^{12}$ W. Ossau, D. Jäkel, E. Bangert, G. Landwehr, and G. Welmann, Surf. Sci. 174, 188 (1986).

${ }^{13}$ Y. Iimura, Y. Segawa, G. E. W. Bauer, M. M. Lin, Y. Aoyagi, and S. Namba, Phys. Rev. B 42, 1478 (1990).

${ }^{14}$ L. Vina, G. E. W. Bauer, M. Potemski, J. C. Maan, E. E.
Mendez, and W. I. Wang, Surf. Sci. 229, 504 (1990).

${ }^{15}$ M. Potemski, L. Viña, G. E. W. Bauer, J. C. Maan, K. Ploog, and G. Weimann, Phys. Rev. B 43, 14707 (1991).

${ }^{16}$ M. Bugajski, W. Kuszko, and K. Reginski, Solid State Commun. 60, 669 (1986).

${ }^{17}$ X. L. Zheng, D. Heiman, and B. Lax, Phys. Rev. B 40, 10523 (1989).

${ }^{18}$ K. J. Nash, M. S. Skolnick, P. A. Claxton, and J. S. Roberts, Phys. Rev. B 39, 10943 (1989).

${ }^{19}$ H. Q. Hou, W. Staguhn, S. Takeyama, N. Miura, Y. Segawa, Y. Aoyagi, and S. Namba, Phys. Rev. B 43, 4152 (1991).

${ }^{20}$ Kyu-Seok Lee, Y. Aoyagi, and T. Sugano, Phys. Rev. B 46, 10269 (1992).

${ }^{21}$ O. Akimoto and H. Hasegawa, J. Phys. Soc. Jpn. 22, 181 (1967).

${ }^{22}$ M. Shinada and K. Tanaka, J. Phys. Soc. Jpn. 29, 1258 (1970).

${ }^{23}$ R. L. Greene and K. K. Bajai, Phys. Rev. B 31, 6498 (1985).

${ }^{24}$ A. H. MacDonald and D. S. Ritchie, Phys. Rev. B 33, 8336 (1986).

${ }^{25}$ S.-R. Eric Yang and L. J. Sham, Phys. Rev. Lett. 58, 2598 (1987).

${ }^{26}$ G. E. W. Bauer and T. Ando, Phys. Rev. B 37, 3130 (1988).

${ }^{27}$ G. E. W. Bauer and T. Ando, Phys. Rev. B 38, 6015 (1988).

${ }^{28}$ Y.-S. Lin, C.-J. Chen, G. Y. Wu, and K.-M. Hung, Solid State Commun. 84, 753 (1992).

${ }^{29}$ J. Kusano, G. E. W. Bauer, and Y. Aoyagi, J. Appl. Phys. 75, 289 (1994). 